\title{
Überlegungen zur Geschichte und Gegenwart der Subjektposition der entwicklungspolitischen (Freiwilligen-)Mobilität
}

\author{
Manuel Peters \\ Dipl. Interkulturelle Pädagogik | Akademischer Mitarbeiter am Fachgebiet Interkulturalität \\ UNESCO Chair in Heritage Studies an der BTU Cottbus-Senftenberg | peters@b-tu.de
}

\section{Zusammenfassung}

Im Beitrag werden Überlegungen zu der spezifischen Zugehörigkeitsposition der entwicklungspolitischen (Freiwilligen-)Mobilität angestellt. Zunächst wird der Frage nachgegangen, was unter Zugehörigkeit genauer zu verstehen ist, um sodann die Zugehörigkeitsposition der (Freiwilligen-)Mobilität historisch zu verorten sowie Kontinuitäten in der Gegenwart aufzuzeigen. Es ergibt sich dann ein Bild der entwicklungspolitischen (Freiwilligen-)Mobilität als eine sich in ihrer Form wandelnde, klassen- und raumbezogene, spezifisch vergeschlechtlichte und rassifizierte Praxis kultureller Distinktion.

Schlagwörter: Zugehörigkeitsordnungen; soziale Zugehörigkeit; Entwicklungszusammenarbeit; Rassismus; Kolonialismus; Intersektionalität; Mobilität, Cultural Studies

\begin{abstract}
This contribution theorizes about (voluntary) development mobility as a specific subject position. To this end, the text aims at clarifying the concept of belonging before it traces histories and continuities of its specific subject positon. Against this background, (voluntary) development mobility appears as changing in form, as well as a class and space specific, gendered, and racialized practice of cultural distinction.
\end{abstract}

Keywords: orders of belonging; social belonging; development cooperation; racism; colonialism; intersectionality, mobility, cultural studies

\section{Kontingente Zugehörigkeitsordnungen und ungleiche Mobilitäten}

Ich möchte mit einem Beispiel aus der deutschen Thematisierung von Migration beginnen, bevor ich zu der Frage komme, wie ich Zugehörigkeit in diesem Beitrag verstehe und warum diese Diskussion für ein Verständnis der entwicklungspolitischen (Freiwilligen-)Mobilität ${ }^{1}$ wichtig ist. Dazu möchte ich mich in aller Kürze der Kategorie „Mensch mit Migrationshintergrund“ zuwenden. Als „Mensch mit Migrationshintergrund“ wurden bis 2011 statistisch alle Menschen gerechnet, die selbst oder deren Vorfahren seit 1949 (bzw. 1955) nach Deutschland migriert sind

1 Ich schreibe von entwicklungspolitischer (Freiwilligen-)Mobilität, weil ich in diesem Text nur bedingt zwischen freiwilliger und anderer entwicklungspolitischer Mobilität unterscheide, obwohl wichtige Unterschiede zwischen den Formen professioneller und freiwilliger Mobilität bestehen. Die Diskussion dieser Unterschiede würde den Rahmen dieses Textes sprengen und erscheint mir gleichzeitig für das hier entwickelte Argument zunächst vernachlässigbar. 
(Statistisches Bundesamt 2013: 26). Dementsprechend wurden auch Menschen der dritten oder vierten Generation ohne eigene Zuwanderungserfahrung aber mit deutschem Pass als Menschen mit Migrationshintergrund erfasst. In offiziellen Statistiken wurde (und wird) Zugehörigkeit zum Kontext Deutschland trotz Staatsangehörigkeit also für eine Vielzahl von Menschen in besonderen Kategorien beschrieben, eine Besonderung, die mit der Überquerung einer nationalstaatlichen Grenze begründet wird und über Generationen hinweg nicht ablegbar scheint. Nun wurde die Definition von „mit Migrationshintergrund“ 2016 geändert, sodass nur noch Menschen, die selbst oder mindestens eines ihrer Elternteile bei Geburt eine andere Staatsbürgerschaft besaßen, in diese Kategorie fallen (Statistisches Bundesamt 2017). Der statistisch festgestellte Zugehörigkeitsunterschied ist also nun, wo er vorher unbegrenzt galt, auf eine bestimmte Zeit begrenzt. Dafür gibt es zahlreiche Gründe und es ließe sich eine lohnenswerte Diskussion der Gründe und der Bedeutung des Wandels anschließen. Mir geht es aber an dieser Stelle zunächst um zwei Dinge. Zum einen zeigt das Beispiel, dass nationalstaatliche Zugehörigkeit offenbar nicht einfach nur eine Angelegenheit des Passes ist, sondern dass die Überquerung einer Grenze irgendwie - in diesem Fall statistisch - nachwirkt. Zum anderen wird deutlich, dass die Definition der Kategorie der nationalstaatlichen Zugehörigkeit Veränderungen unterworfen ist und (nicht nur) der deutsche Zugehörigkeitskontext von sich verändernden Ordnungen der Zugehörigkeit geprägt ist. Es ist also offensichtlich notwendig, nationalstaatliche Zugehörigkeit als eine kontingente und das heißt nicht so und nicht in dieser Art und Weise notwendige Kategorie zu verstehen, was es bedeutsam macht, Verständnisse von Zugehörigkeit und ihre Herstellung genauer zu betrachten.

Das einführende Beispiel der Klassifizierung von Migration ist auch weiter gehend für die hier beabsichtigte Diskussion von entwicklungspolitischer (Freiwilligen-) Mobilität bedeutsam, weil wichtige Unterschiede zwischen verschiedenen Formen der räumlichen Bewegung von Menschen und ihrer Klassifizierung bestehen. Während einigen grenzüberschreitenden Bewegungen von Menschen nur wenig Widerstand begegnet, sie einfach nur mobil sind und sie dabei nur kaum als Migrant*innen wahrgenommen werden, stoßen andere Menschen auf größere Widerstände, was sich auch in ihrer Kategorisierung als Migrant*innen ausdrückt (Bauman 1998; Amelina 2017). Unterschiedlich bewertete Mobilitäten erscheinen in der Folge als gesellschaftlich ausgehandelte Varianten von Mobilität, deren veränderlicher Status eine Frage von historischen und aktuellen Machtverhältnissen ist (Manderscheid 2012). Meza Torres (2012: 217) weist in diesem Zusammenhang auf die Bedeutung der Rassifizierung der Kategorie des*r Immigranten*in hin. Diejenigen, die auf Widerstände stoßen, die als Immigrant*innen gelten und/oder die ihren Migrationsstatus auch über mehrere Generationen hinweg nicht loswerden, sind ihr zufolge die ehemaligen „colonial/racial subjects of empire“, auf deren Grundlage die Vorherrschaft der „westlichen“Welt zur Zeit des Kolonialismus aufgebaut wurde, und die sich nun im 
Inneren des ehemaligen Imperiums befinden (ähnlich auch Balibar 2009). Die Wissensbestände des ehemals kolonialen Zentrums sind aus dieser Perspektive auch weiterhin von kolonial-rassistischem Wissen geprägt und weiterhin in dessen Zugehörigkeitskonzepte eingeschrieben. Diesen Befund bestätigt auch die rassismuskritisch informierte deutsche Migrationsforschung (Mecheril 2003; Rose 2011; Scharathow 2014; Velho 2015), die aufzeigt, dass und wie die Zugehörigkeitskonzepte des Kontextes Deutschland systematisch von Rassismus geprägt sind. Vor dem Hintergrund dieser Diskussion erscheint die entwicklungspolitische Mobilität in einem Zugehörigkeitsraum spezifisch positioniert, der sich vor dem Hintergrund von historischen und aktuellen Machtverhältnissen konstituiert. Dass sie als Mobilität und nicht als Migration markiert ist, weist dabei auf ihren privilegierten Status hin.

Bevor ich mich genauer mit der spezifischen Position der entwicklungspolitischen Mobilität zuwende, möchte ich zunächst genauer klären, was unter sozialer Zugehörigkeit als nicht an sich gegebene, sondern veränderliche Kategorie eigentlich zu verstehen ist.

\section{Zugehörigkeitskonzepte, -erfahrungen und -profile}

Werden Kategorien sozialer Zugehörigkeit (s. u.) nicht als an sich gegeben, sondern als der Herstellung bedürftig verstanden, rücken Praktiken der Klassifikation und Bezeichnung von Zugehörigkeit in den Blick, also die Frage danach, wie es kommt, dass Zugehörigkeit jeweils so und nicht anders verstanden wird. Dabei lassen sich drei Ebenen unterscheiden (Mecheril 2003: 127 ff.; Mecheril 2018):

1. Zunächst die - diskursive - Ebene der Zugehörigkeitskonzepte: Zugehörigkeitskonzepte definieren den Rahmen und damit auch die Grenzen von Zugehörigkeit, das heißt „was Zugehörigkeit ist, wer zugehörig sein darf, welche Rechte und Pflichten mit Zugehörigkeit einhergehen, wie Loyalitätsbrüche sanktioniert werden und [...] in welcher Weise über Zugehörigkeitsansprüche verhandelt und entschieden wird“ (Mecheril 2003: 128). Um Zugehörigkeitskonzepte wird politisch, gesellschaftlich und intersubjektiv gestritten, sie sind Ausdruck umstrittener und wandelbarer Zugehörigkeitsdiskurse (Wallerstein 1991: 77; Hall 2006: 221). In Hinsicht auf Auseinandersetzungen um die Art der Ausgestaltung von Zugehörigkeitskonzepten haben die Akteur*innen unterschiedliche Möglichkeiten „ihre Konzeption ,guter Zugehörigkeitsverhältnisse' juristisch, politisch oder alltagsweltlich wirksam werden zu lassen“ (Mecheril 2003: 129). Zugehörigkeitskonzepte sind Ausdruck von Macht- und Kräfteverhältnissen und ihre genaue Ausgestaltung unterscheidet sich damit je nach Akteur*innen, Kontext und Zeit. Gemeinsam ist dabei allen Zugehörigkeitskonzepten, dass sie eine Differenz zwischen Wir und den Anderen instituieren, denjenigen, die von Zugehörigkeitskonzepten als dazugehörig eingeschlossen sind und denjenigen, die außerhalb positioniert werden (Sutherland 
2014). Zugehörigkeitskonzepte sind deshalb immer relational (Mecheril 2003: 119). Als prominente Kategorien sozialer Zugehörigkeit gelten „Rasse“, Klasse, Geschlecht, Ethnizität, Alter, aber auch bspw. Raum (Marchart 2008; Wallerstein 1991; Degele/Winker 2009; Amelina 2017). In ihrer - intersektionalen - Verschränkung ergeben sich spezifische subjektive Zugehörigkeitspositionierungen und werden Subjekten bestimmte Zugehörigkeitserfahrungen nahegelegt. Zugehörigkeitskonzepte bestimmen insofern den Rahmen, innerhalb dessen individuelle Zugehörigkeitserfahrung in verschiedenen Variationen möglich wird.

2. Des Weiteren die Ebene der Zugehörigkeitserfahrungen: Zugehörigkeitserfahrungen werden in sozialen Zusammenhängen gemacht, welche von bestimmten Zugehörigkeitskonzepten geprägt sind. Diese legen bestimmte Möglichkeiten der Erfahrung von Zugehörigkeit nahe. Diese Erfahrungen können sich sowohl positiv als auch negativ in Bezug auf (nicht) dazugehören gestalten und finden vor dem Hintergrund von Fremd- als auch Selbstzuschreibungen statt, wobei Fremd- und Selbstzuschreibung auch konfligieren können. Zugehörigkeitserfahrungen sind dann idealtypisch zu verstehen als Anerkennungs- oder Ablehnungserfahrungen, in denen der*die Einzelne als zugehörig oder nicht zugehörig „(an)erkannt“ wird und/oder sich selbst als zugehörig oder nicht zugehörig „(an)erkennt“ (Mecheril 2003: 130), sich also zu Zugehörigkeitskonzepten positioniert und diesbezüglich positioniert wird. Zugehörigkeitskonzepte lassen sich deshalb auch als „Subjektpositionen" (Spies 2017: 72) verstehen, die dann von Subjekten in Prozessen der Fremd- und Selbstidentifikation eingenommen werden. Subjekte besitzen durchaus Gestaltungsspielraum in Hinsicht auf Zuschreibungen von Zugehörigkeit, auch, da kein Konzept völlig eindeutig sein kann und weil niemals nur ein Zugehörigkeitskonzept von Bedeutung ist, das heißt, in der Intersektion mit anderen Konzepten Spielraum besteht (Spies 2017: 71).

3. Zuletzt die Ebene der subjektiven Zugehörigkeitsverständnisse: Zugehörigkeitsverständnisse können sich von Zugehörigkeitserfahrungen und Zugehörigkeitskonzepten unterscheiden, weil sie Ergebnis der jeweiligen Geschichte und Gegenwart gemachter positiver wie negativer Zugehörigkeitserfahrungen sind. Dies lässt sich auch als subjektive Bildungsgeschichte in Hinsicht auf Zugehörigkeitskonzepte verstehen, über die Subjekte „übergeordnete Strukturen der Kenntnis, des Handelns und Befindens" (Mecheril 2003: 132) ihre eigene Zugehörigkeit und die Zugehörigkeit anderer betreffend entwickeln. Diese bilden den Rahmen für die jeweils individuelle kontextspezifische Zugehörigkeitspraxis und sind kontext- und erfahrungsabhängig. Verständnisse des*r Einzelnen und seiner*ihrer Umwelt lassen sich also nicht voneinander trennen und Subjekte müssen als dezentrierte Subjekte verstanden werden, als „Subjekt[e]-imWerdegang“ (Spies 2017: 78, zitiert nach Hall 2004: 176). 
Die obenstehenden Erläuterungen verdeutlichen, dass subjektive Zugehörigkeitserfahrungen im Kontext bestimmter vorherrschender Zugehörigkeitskonzepte ihren Sinn gewinnen. Diese sind historisch und geographisch verschieden und kontingent sowie Gegenstand von Auseinandersetzungen, in die Akteur*innen aus sehr unterschiedlichen Zugehörigkeitspositionen heraus eintreten und also unterschiedliche Möglichkeiten mitbringen, diese zu beeinflussen. Subjektive Zugehörigkeitsverständnisse, die als (unabgeschlossenes und veränderliches) Ergebnis der gemachten Erfahrungen von Zugehörigkeit zu verstehen sind, können sich nun von Zugehörigkeitskonzepten unterscheiden, das heißt, Fremd- und Selbstzuschreibung von Zugehörigkeit müssen nicht übereinstimmen. Zugehörigkeitskonzepte bilden aber den allgemeinen Rahmen, der bestimmten Zuschreibungen mehr oder weniger gesellschaftliche Legitimität verleiht.

Im Folgenden möchte ich nun genauer auf die spezifische Subjekt- bzw. Zugehörigkeitsposition der entwicklungspolitischen Mobilität eingehen. Zu diesem Zweck werde ich einen genaueren Blick auf ihre allgemeine Rahmung werfen, auf Zugehörigkeitskonzepte, wie sie für die entwicklungspolitische (Freiwilligen-)Mobilität relevant erscheinen. Aus Platzgründen beschränke ich mich dabei auf die Ebene der Zugehörigkeitskonzepte. Es ist vor dem Hintergrund der vorherigen Ausführungen davon auszugehen, dass Zugehörigkeitskonzepte etwas über den möglichen Rahmen der Zugehörigkeitserfahrungen aussagen, die den jungen Erwachsenen im Weltwärts-Programm begegnen und die sie hervorbringen. Allerdings gilt dies auch umgekehrt, wenn nicht sogar noch mehr: aus Zugehörigkeitserfahrungen lässt sich genaueres über die jeweils geltenden Zugehörigkeitskonzepte, ihre (kontextuelle) Aktualität und ihren Wandel sowie über ihre jeweilige konkrete subjektive Bearbeitung lernen. Dazu Spies:

Positionierungen sind als „Lesarten“ eines Diskurses zu verstehen und geben damit Hinweise darauf, welche Diskurse derzeit dominant sind und keine alternative Lesart zulassen und welche Diskurse zwar eine bestimmte Bedeutung nahelegen, das Bedeutete aber nicht festhalten können und damit einen Spielraum bei der Positionierung des*der Einzelnen ermöglichen (Spies 2017: 84).

\section{Zur Subjektposition der entwicklungspolitischen Mobilität - genealogische Perspektiven}

Barbara Heron (2007) beschäftigt sich mit der Genealogie der Subjektposition des “development workers". Für Heron geht diese Subjektposition zurück auf die Herausbildung der europäischen bürgerlichen Subjektform im 18. und 19. Jahrhundert. Für diese Zeit war (nicht nur) in Europa die Bildung von Nationalstaaten, das Projekt der Aufklärung, Kolonialismus sowie die industrielle Revolution wesentlich (Heron 2007: 29ff.). Die sich herausbildende bürgerliche Subjektform war eng mit diesen Prozessen verwoben, ermöglichte sie und wurde durch sie ermöglicht. Es ist diese Zeit, in der sie sowohl kulturell wie ökonomisch zunehmend hegemonial 
wird (Reckwitz 2006: 249). Zur Legitimierung ihrer Hegemonie diente vor allem ein „Gegensatz zwischen Moralischem und Amoralischem“ (Reckwitz 2006: 249). So positioniert sich das bürgerliche Subjekt zunächst im 18. Jahrhundert in Abgrenzung gegenüber einer als verfälscht, unvernünftig und unnatürlich markierten Aristokratie. Im 19. Jahrhundert wird Zivilisiertheit zum moralischen Leitschema und bürgerliche Subjektivität im Gegensatz zu "that which was uncivilized, lower class, and non-European" (Heron 2007: 29, zitiert nach Stoler 2000: 151) artikuliert. Diese Differenz zwischen Zivilisiertheit und Unzivilisiertheit ist in starkem Maße hierarchisierend und stellt Höher- und Minderwertigkeiten fest, die durch spezifische kulturelle Kompetenzen, spezifisches Wissen, spezifische Werte und spezifische Sensibilitäten nach außen demonstriert werden müssen und in denen sich bürgerliche Subjekte in der Folge einübten (Heron 2007: 29; Reckwitz 2006: 242 ff.) Diese Praktiken kultureller (und rassischer) Distinktion waren in hohem Maße auf die Präsenz zivilisatorisch nicht gleichwertig konstruierter ,Anderer" angewiesen. ${ }^{2}$ Dies waren unter anderem "the working classes, the colonized, women, gypsies, Jews, the Irish, and criminals" (Heron 2007: 29). Die enge Verbindung bourgeoiser Subjektivität und die Einübung einer spezifischen Form moralischer Regulierung und kultureller Kompetenzen führte darüber hinaus zu der Vorstellung, dass den verschiedenen ,Anderen' nicht zugetraut werden konnte, sich selbst zu regieren. Sie wurden vielmehr als der Zivilisierung durch Regierung und der Erziehung in der ,richtigen' Art zu leben bedürftig angesehen, ohne dabei jemals ganz so kultiviert werden zu können, wie die bürgerlichen Subjekte selbst (Heron 2007: 30, Reckwitz 2006: 251). Dieses Verständnis bürgerlicher Subjektivität erklärt den Eingriff in die Leben anderer sozialer Gruppen für notwendig und legitim, sogar zu einer Verantwortung, die als „white mans burden“ (Piller 2011: 21) bekannt geworden ist. In der bürgerlichen Subjektform kommt also eine spezifische Klasse zum Ausdruck, die zwischen Proletariat und Aristokratie angesiedelt war und zunehmend die Vormachtstellung innehatte. Zentral für diese Klassenkonstitution erscheinen Praktiken kultureller Distinktion gegenüber als rückständig imaginierten Klassen und „Rassen“. Darüber hinaus brachte bürgerliche Subjektivität auch eine spezifische Form von Männlichkeit und Weiblichkeit hervor und zwar zunächst ,entlang der Codes von, öffentlicher' Männlichkeit und ,familiärer' Weiblichkeit“" (Reckwitz 2006: 243; Heron 2007: 31). Die Rolle der Ausbildung erkennbar angemessener, bürgerlich kultureller Kompetenzen und Subjekte fiel damit vornehmlich der weiblichen Position zu, während die Zivilisierung und Erziehung der ,Anderen` zunächst eine männliche Domäne war. Weibliche Subjektposition bedeutete dann, auf der einen Seite Hüterin und Erzieherin von Moral und Charakter zu sein. Auf der anderen Seite war Weiblichkeit aber

Rassismus ist nicht nur als biologischer Rassismus zu verstehen, sondern wie am Beispiel des „new racism“ deutlich wird, dem Kultur als Synonym für „Rasse“ dient, als wandelbar und potentiell eine ganze Reihe kultureller, biologischer und/oder religiöser Referenzen als Marker „rassischer“ Differenz heranziehend und miteinander artikulierend (Balibar/ Wallerstein 1991; Balibar 2009; Lentin 2008: 489). 
auch durch ,Emotionalität‘, ,Unvernunft' und ,suspekte Sexualität‘ markiert und damit teilweise auf der Seite der Unkultiviertheit positioniert. Das machte das Konzept bürgerlicher Weiblichkeit im Gegensatz zu bürgerlicher Männlichkeit zu einer ambiguen Position, da Zuschreibungen von Unvernunft und Unkontrolliertheit als Synonyme der Unzivilisiertheit auch aufseiten des*r , unkultivierten' und/oder, rassifizierten',Anderen“ verortet wurden (und werden) (Heron 2007: 30). Diese ambigue Position konnte nur durch vorzeigbare und gute moralische Lebensweise und gute Sitten sowie intergenerationale Weitergabe von bürgerlicher Sensibilität und Moral ausgeglichen werden, „to the extent that the moral became the purview and domain of white womanhood“ (Heron 2007: 31). Folgende Kämpfe um mehr Partizipation im öffentlichen Raum führten zu Verschiebungen der geschlechtlichen Zugehörigkeitskonzepte und zunächst dazu, dass öffentliche Sphären, in denen wohltätiges Engagement gefragt war, zunehmend weiblich dominiert waren, weil diese Bereiche mit vorherrschenden Vorstellungen bürgerlicher Weiblichkeit ${ }^{3}$ am ehesten in Einklang zu bringen waren. Das Engagement von bürgerlichen Frauen in öffentlichen Bereichen galt zunehmend nicht mehr nur als angemessen, sondern wurde auch zu einer Verpflichtung, um in einem ,guten' Lichte zu erscheinen (Heron 2007: 32). Es ist einleuchtend, dass missionarische Arbeit in diesem Zusammenhang ebenfalls an Bedeutung gewann. Weitere Kämpfe und korrespondierende Diskursverschiebungen führten dazu, dass in den letzten 30 Jahren des 19. Jahrhunderts Frauen die Mehrheit in der missionarischen Tätigkeit in den Kolonien stellte (Heron 2007: 32).

\section{Zur Subjektposition der entwicklungspolitischen Mobilität - Kontinuitäten und Anknüpfungspunkte}

Vor dem Hintergrund der zuvor geführten Diskussion stellt sich die Frage, ob und wie sich die Geschichte der bürgerlichen Zugehörigkeitskonzepte und ihre Verbindung mit dem „Entwicklungsprojekt“ in der heutigen entwicklungspolitischen (Freiwilligen-)Mobilität wiederfindet.

Moralität bleibt Heron (2007: 34) zufolge auch heute prägend für die Identität als (weißes) bürgerliches Subjekt, wenn auch in abgewandelter Form. Anstelle des Zivilisierungsdiskurses ist das „Prisma“ eines „globalen Bewusstseins“ getreten, durch das ein moralisches Subjekt konstruiert wird, das den Anspruch und die Verantwortung fühlt, anderen, helfen' zu müssen.

Dabei sind nicht globales Bewusstsein und Verantwortung problematisch, sondern eine weiterbestehende rassifizierte und rassifizierende Konstruktion des Verständnisses von Bewusstsein und Verantwortung, eine Verantwortung, die die

3 Es ist wichtig, darauf hinzuweisen, dass es hier um die Zugehörigkeitskonzeption einer bestimmten Klasse, der bürgerlichen Klasse geht. In anderen Klassen können auch andere Konzeptionen vorherrschen. Die zu findende Fokussierung auf Weiblichkeit geschieht hier aus Platzgründen und weil drei Viertel der Teilnehmer*innen im Weltwärts-Programm Frauen sind (s. u.). 
relationalen Bezüge der moralischen Konstitution der bürgerlichen Subjektform (zur Geschichte des Kolonialismus) weiterhin weitgehend ausblendet. Die Mobilitäten im Weltwärts-Programm finden bspw. vor dem Hintergrund bereits existierender (und) medial vermittelter Bilder über die Partnerländer und -regionen statt. Dieses Wissen bspw. über „indigenes Leben“, „Afrika“, „Lateinamerika“ muss gewissermaßen nur noch abgerufen werden (Kontzi 2015: 201). Wie nicht zuletzt der Film „white charity“ (Kiesel/Phillipp: 2012) zeigen kann, wird die Welt dabei weiterhin in rassifizierter Form räumlich repräsentiert.

\section{[...] the countries of the North - home to the former metropoles of empire and their white-settler dominions such as Canada [...] places of greater civiliza- tion, of order, cleanliness and a truly good quality of life, which has an evident material basis of comfort and security, while those of the South - the former colonies - languish in an anachronistic space, where chaos often reigns, disor- der and disease are rampant and life seems (from our perspective) to be hardly worth living (Heron 2007: 34).}

Die Art der Repräsentation von Raum führt dabei neben der offensichtlichen Rassifizierung und Kulturalisierung ,der Anderen' auch zur Selbstaffirmation "through comparative racialized discourse" (Heron 2006: 35). Diese Art der rassifizierenden Selbstbestätigung lässt sich in Kontinuität zur Geschichte der bürgerlicher Subjektform setzen (Huffer 2018: 34 f.). Heron (2007: 37) erscheint es für die Wirksamkeit dieses Diskurses über Raum unwesentlich, wie viele Menschen sich im Entwicklungsprojekt wirklich engagieren, so lange die „Entwicklungsunternehmung“ an sich weitergeht. In diesem Sinne argumentiert Huffer (2018: 34 ff.), dass die diskursive Verortung von Freiwilligendiensten als entwicklungspolitisch weitgehende Konsequenzen für Raumverständnisse hat. So sind mit dieser Verortung auch die geographischen Orte definiert, in und zwischen denen sich die Freiwilligenmobilität abspielt, und zwar zwischen als „unterentwickelt“ und „entwickelt" klassifizierten geographischen Kontexten (Huffer 2018: 35 f.).

Nach Heron (2007: 37) ist auch die Geschichte der Vergeschlechtlichung bürgerlicher Subjektivität, das heißt ,angemessener' Weiblichkeit und Männlichkeit weiterhin bedeutsam. Sie argumentiert, dass vor dem Hintergrund der Geschichte der bürgerlichen Subjektposition eine spezifische, eventuell stärkere, Verbindung zwischen „white middle class women“ und Entwicklungszusammenarbeit besteht, als dies bei „male bourgeois subjects“ der Fall ist, was ihr zufolge auch zu einer stärkeren Präsenz von Frauen im Entwicklungsprojekt führt. Dieser Befund lässt sich für das Beispiel der Weltwärts-Mobilität zunächst bestätigen. So waren 2017 bspw. 69,6 Prozent der Freiwilligen Frauen (weltwärts o. J.). Entwicklungspolitische Freiwilligendienste bleiben darüber hinaus eine klassenbezogene Angelegenheit, sie waren und sind wenig inklusiv, sondern erscheinen als Programme der bürgerlichen Mittel- und Oberschicht (Schmidt 2013). Kontzi (2015: 113) bringt dies wie folgt auf 
den Punkt: der*die typische Weltwärts-Freiwillige ist eine ,junge Frau [...] Abiturientin, von Anfang 20 mit deutscher Staatsangehörigkeit und ohne anerkannte Behinderung [...] aus einer Akademiker_innenfamilie, in der vorwiegend deutsch gesprochen wird, die ein hohes Erwerbseinkommen hat und im Westen Deutschlands lebt“.

Die Subjektposition der (freiwilligen) „Entwicklungszusammenarbeit“ erscheint also klassenspezifisch und spezifisch vergeschlechtlicht und, neben der kulturellen Abgrenzung von anderen Klassen, auf rassifizierte Vorstellungen von Raum angewiesen. Dies zeigt die genealogische Perspektive auf Zugehörigkeitskonzepte. Sie zeigt ebenfalls, dass sich die Konzepte einerseits gewandelt haben und dass sie andererseits weiterhin deutliche Spuren ihrer Geschichte tragen.

Ob und wie genau (bspw. mit welchen Praktiken der Distinktion, die mit was für Zugehörigkeitserfahrungen und -verständnissen einhergehen) die Subjektposition der entwicklungspolitischen (Freiwilligen)Mobilität in der und durch die Entwicklungsunternehmung reproduziert und verändert wird, kann ein Blick auf das Zusammenspiel von Zugehörigkeitskonzepten, individuellen Zugehörigkeitserfahrungen und Zugehörigkeitsverständnissen besser verständlich machen.

\section{Literaturverzeichnis}

Amelina, Anna (2017): Transnationalizing inequalities in Europe. Sociocultural boundaries, assemblages and regimes of intersection, New York-London.

Balibar, Etienne (2009): Europe as Borderland, in: Environment and Planning D Society and Space, Jg. 27, Heft 2, S. 190-215.

Bauman, Zygmunt (1998): Globalization. The Human Consequences, Cambridge.

Degele, Nina und Gabriele Winker (2009): Intersektionalität. Zur Analyse sozialer Ungleichheiten, 2. Aufl., Bielefeld.

Hall, Stuart (2008): Whose heritage? Un-settling 'the heritage', Re-Imagining the Post-Nation, in: The Heritage Reader, edited by Graham Fairclough, Rodney Harrison, John Schofield and John H. Jameson, London, S. 219-228.

Hall, Stuart (2004): Wer braucht Identität?, in: Stuart Hall. Ideologie, Identität, Repräsentation. Ausgewählte Schriften 4, hrsg. von Juha Koivisto und Andreas Merkens, Hamburg, S. 167-187.

Heron, Barbara (2007): Desire for development. Whiteness, Gender, and the Helping Imperative, Waterloo, Ontario.

Huffer, Matthias (2018): Das Andere im internationalen Freiwilligendienst. Rassismus. Subjektivität. Verantwortung, in: Voluntaris Jg. 6, Heft 1, S. 29-43.

Kiesel, Timo und Carolin Phillipp (2012): White Charity - Schwarzsein \& Weißsein auf Spendenplakaten. Dokumentarfilm, 48 Minuten.

Kontzi, Kristina (2015): Postkoloniale Perspektiven auf „weltwärts“: Ein Freiwilligendienst in weltbürgerlicher Absicht, Lüneburg.

Lentin, Alana (2008): Europe and the Silence about Race, in: European Journal of Social Theory, vol. 1 , no. 4 , S. 487-503. 
Manderscheid, Katharina (2012): Mobilität, in: Handbuch Stadtsoziologie, hrsg. von Frank Eckardt, Wiesbaden, S. 551-570.

Marchart, Oliver (2008): Cultural studies, Konstanz.

Mecheril, Paul (2003): Prekäre Verhältnisse. Über natio-ethno-kulturelle (Mehrfach-)Zugehörigkeit, Münster.

Mecheril, Paul (2018): Was meint soziale Zugehörigkeit?, in: Identität in der modernen Arbeitswelt: neue Konzepte für Zugehörigkeit, Zusammenarbeit und Führung, hrsg. von Olaf Geramanis und Stefan Hutmacher, Wiesbaden, S. 21-32.

Meza Torres, Andrea (2012): Die Cité nationale de l'histoire de l'immigration und die „Immigranten“ im Kontext der Kolonialgeschichte. Interaktionen und politische Interventionen, in: Museum und Migration: Konzepte - Kontexte - Kontroversen, hrsg. von Regina Wonisch und Thomas Hübel, Bielefeld, S. 193-222.

Piller, Ingrid (2011): Intercultural Communication. A Critical Introduction, Edinburgh.

Reckwitz, Andreas (2006): Das hybride Subjekt. Eine Theorie der Subjektkulturen von der bürgerlichen Moderne zur Postmoderne, Weilerswist.

Rose, Nadine (2011): Migration als Bildungsherausforderung: Subjektivierung und Diskriminierung im Spiegel von Migrationsbiographien , Bielefeld.

Scharathow, Wiebke (2014): Risiken des Widerstandes. Jugendliche und ihre Rassismuserfahrungen, Bielefeld.

Schmidt, Nicole D. (2013): Freiwilligendienste - Eine exklusive Veranstaltung?, in: Voluntaris, Jg. 1, Heft 2, S. 101-109.

Spies, Tina (2017): Subjektpositionen und Positionierungen im Diskurs, in: Biographie und Diskurs: Methodisches Vorgehen und Methodologische Verbindungen, hrsg. von Tina Spies und Elisabeth Tuider, Wiesbaden, S. 69-90.

Spies, Tina und Elisabeth Tuider (Hrsg.) (2017): Biographie und Diskurs: Methodisches Vorgehen und Methodologische Verbindungen, Wiesbaden.

Statistisches Bundesamt (2013): Zensus 2011: Ausgewählte Ergebnisse, Wiesbaden.

Statistisches Bundesamt (2017): Fachserie 1, Reihe 2.2 Bevölkerung und Erwerbstätigkeit, Bevölkerung mit Migrationshintergrund, Ergebnisse des Mikrozensus, Wiesbaden.

Stoler, Ann Laura (2000): Race and the education of desire. Foucault's history of sexuality and the colonial order of things, 5. Aufl., Durham.

Sutherland, Claire (2014): Leaving and Longing: Migration Museums as Nation-Building Sites, in: Museum and Society, vol. 12, no. 2, S. 118-142.

Velho, Astride (2015): Alltagsrassismus erfahren. Prozesse der Subjektbildung - Potenziale der Transformation, Frankfurt a. M.

Weltwärts (o. J.): Aktuelle Zahlen und Fakten rund um das Programm weltwärts Entsendeorganisationen, www.weltwaerts.de/de/presse.html (07.06.2019).

Wallerstein, Immanuel Maurice (1991): The Construction of Peoplehood: Racism, Nationalism, Ethnicity, in: Race, Nation, Class. Ambiguous Identities, edited by Étienne Balibar und Immanuel Maurice Wallerstein, London-New York, S. 71-85. 